\title{
Undecidability of the existence of dictator for strongly candidate stable voting procedures in an infinite society and Cantor's diagonal argument
}

\author{
YASUHITO TANAKA \\ Faculty of Economics, Doshisha University, Kamigyo-ku, Kyoto, 602-8580, Japan \\ E-mail: yatanaka@mail.doshisha.ac.jp
}

\begin{abstract}
The strong candidate stability theorem by Dutta et al. [4], one of the major theorems of social choice theory, states that, with a finite number of voters, there exists a dictator for any voting procedure which satisfies strong candidate stability, strong unanimity and independence of irrelevant alternatives (IIA). This paper investigates a decidability problem of voting procedures in a society with an infinite number of individuals (infinite society) using Cantor's diagonal argument presented by Yanofsky [19] which is based on Lawvere [10]. We will show the following result. The problem whether a strongly candidate stable voting procedure has a dictator or has no dictator in an infinite society is undecidable. It is proved using the arguments similar to those used to prove an extended version of Cantor's theorem that there cannot be an onto function from $\mathbb{N}$ (the set of natural numbers) to its power set $\mathcal{P}(\mathbb{N})$. This undecidability means that for any strongly candidate stable voting procedure we can not decide whether or not it has a dictator in finite steps by some program. A dictator of a voting procedure is a voter such that if he strictly prefers a candidate (denoted by $x$ ) to another candidate (denoted by $y$ ), then the voting procedure does not choose $y$. Strong candidate stability requires that there be no change in the outcome of an election if a candidate withdraws who would lose if every candidate stood for office.
\end{abstract}

Mathematical subject classification: $91 \mathrm{~B} 12,91 \mathrm{~B} 14,16 \mathrm{~B} 50$.

Key words: strongly candidate stable voting procedure, Cantor's diagonal argument. 


\section{Introduction}

This paper investigates a decidability problem of an extension of the strong candidate stability theorem by Dutta et al. [4] to a society with an infinite number of individuals (infinite society) using Cantor's diagonal argument presented by Yanofsky [19] which is based on Lawvere [10] ${ }^{1}$. According to [19] an extended version of Cantor's theorem is stated as follows.

Let $Y$ be a set, and $\alpha: Y \rightarrow Y$ a function without a fixed point (for all $y \in Y, \alpha(y) \neq y), T$ and $S$ sets and $\beta: T \rightarrow S$ a function that is onto (i.e., has a right inverse $\bar{\beta}: S \rightarrow T$ ), then for all functions $f: T \times S \rightarrow Y$ the function $g: T \rightarrow Y$ constructed as follows

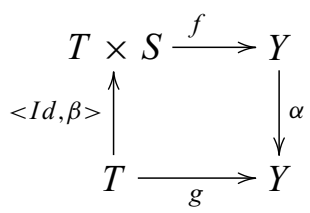

is not representable by $f$. Where $I d$ is the identity mapping on $T$.

It is an extension of the famous Cantor's theorem that the cardinality of the power set $\mathcal{P}(\mathbb{N})$ of the set of natural numbers $\mathbb{N}$ is larger than the cardinality of $\mathbb{N}$, or in other words, there is no onto map $\mathbb{N} \rightarrow \mathcal{P}(\mathbb{N})$. We will use similar arguments to those in the proof of this theorem to prove our main result.

The strong candidate stability theorem by Dutta et al. [4] is one of major theorems of social choice theory. About social welfare function (binary social choice rule which satisfies some conditions), Arrow's impossibility theorem ([1]) states that, with a finite number of individuals, there exists a dictator for

\footnotetext{
${ }^{1}$ In other papers we examined computability or decidability of social choice rules. For example, in [17], we examined social choice function from the point of view of constructive mathematics, and showed that the theorem that any coalitionally strategy-proof social choice function may have a dictator or has no dictator in an infinite society is equivalent to LPO (Limited principle of omniscience). About LPO, see [3]. A social choice function is a social choice rule that chooses one alternative from the set of alternatives about some issue corresponding to reported profiles of individual preferences in a society. If any group (finite or infinite) of individuals has no incentive to report falsely the preferences of its members, then the social choice function is coalitionally strategy-proof. Coalitional strategy-proofness is an extension of the ordinary strategy-proofness. It requires non-manipulability by coalitions of individuals as well as by a single individual.
} 
any social welfare function. In contrast [6], [8] and [9] showed that when the number of individuals in a society is infinite, there exists a social welfare function without dictator. About strategy-proof social choice functions, with a finite number of individuals, the Gibbard-Satterthwaite theorem ([7] and [14]) states that there exists a dictator for any strategy proof social choice function. In contrast [13] showed that in an infinite society there exists a coalitionally strategy proof social choice function without dictator ${ }^{2}$. About strongly candidate stable voting procedures [4] showed that, with a finite number of voters, there exists a dictator for any voting procedure which satisfies strong candidate stability, strong unanimity and independence of irrelevant alternatives (IIA). We will show that there may be no dictator for any strongly candidate stable voting procedure satisfying strong unanimity and IIA in an infinite society. A dictator of a voting procedure is a voter such that if he strictly prefers a candidate (denoted by $x$ ) to another candidate (denoted by $y$ ), then the voting procedure does not choose $y$. Strong candidate stability requires that there be no change in the outcome of an election if a candidate withdraws who would lose if every candidate stood for office.

In the next section we present the framework of this paper and some preliminary results. In Section 3 we will show the following result. The problem whether a strongly candidate stable voting procedure has a dictator or has no dictator in an infinite society is undecidable. And it is proved using the arguments similar to those used to prove an extended version of Cantor's theorem. This undecidability means that for any strongly candidate stable voting procedure we can not decide whether or not it has a dictator in finite steps by some program.

\section{The framework and preliminary results}

About formulation of voting procedures we refer to [5]. Let $C$ be the finite set of potential $m$ candidates with $m \geq 3$. The set of all subsets of $C$ is denoted by $C$. The candidates are represented by $x, y, z$ and so on, or by $x_{1}, x_{2}, \cdots, x_{m}$. $V$ is the set of a countably infinite number of voters. $C$ and $V$ may overlap, that is, some candidates may also be voters. Let $C_{1}$ be the set of voting candidates and $C_{2}$ be the set of non-voting candidates. Thus $C_{1} \cup C_{2}=C, C_{2} \cap V=\emptyset$ and

\footnotetext{
${ }^{2}[18]$ is a recent book that discusses social choice problems in an infinite society.
} 
$C_{1} \subset V$. Since $V$ is an infinite set, there exist voters who are not candidates. Each individual (voter or candidate) $i$ is endowed with a preference ordering $\succ_{i}$ on $C$. Individual preferences over the candidates are transitive weak orders, that is, they prefer a candidate to another candidate, or are indifferent between them. Since there are a finite number of candidates, the varieties of weak orders over the candidates are finite. We denote the set of individual preferences by $\Sigma$, and denote $x \succ_{i} y$ when individual $i$ prefers $x$ to $y, x \sim_{i} y$ when he is indifferent between them.

A combination of individual preferences is called a profile, and it is denoted by $p, p^{\prime}$ and so on. For each $i p$ assigns a preference ordering $\succ_{i}, p^{\prime}$ assigns a preference ordering $\succ_{i}^{\prime}$ and so on. Given $\succ_{i}$ we denote by $\left.\succ_{i}\right|_{S}$ the restriction of $\succ_{i}$ to $S \subseteq C$. Similarly, $\left.p\right|_{S}$ denotes the restriction of a profile $p$ to the set $S$. The set of profiles is denoted by $\Sigma^{\omega}$, where $\omega=\{1,2, \cdots\}$ denotes the set of natural numbers. $\omega$ represents the set of voters.

We consider a voting procedure $v:\left\{\Sigma^{\omega}, C\right\} \longrightarrow C$. It is defined over subsets of $C$. But it may not be defined over all subsets of $C$. A subset of $C$ for which a voting procedure is defined is called a feasible set. We denote the set of all feasible sets by $\mathcal{B}$. $\mathcal{B}$ is a subset of $C$. Further we assume that any voting procedure is defined for $B \in C$ such that $|B|=m-1$ or $|B|=m$, where $|B|$ denotes the number of candidates in $B$. We consider a single-valued voting procedure, that is, it chooses at most one and at least one candidate from $B$ at every profile. Given profiles $p, p^{\prime} \ldots$ and sets in $\mathcal{B} B, B^{\prime} \ldots$ we denote by $v(p, B), v\left(p^{\prime}, B\right), v\left(p, B^{\prime}\right), v\left(p^{\prime}, B^{\prime}\right) \ldots$ the candidate chosen by a voting procedure at each profile from a feasible set.

The set of top-ranked (or most preferred) candidates in $B$ according to $\succ_{i}$ is denoted by

$$
T\left(B, \succ_{i}\right)=\left\{x \mid x \in B \text {, and } x \succ_{i} y \text { for all } y \in B \backslash T\left(B, \succ_{i}\right)\right\}
$$

Every voting candidate's unique top-ranked candidate is usually himself. Thus, the domain of voting procedures is restricted to the following set.

$$
\Sigma^{\omega}(r)=\left\{p \in \Sigma^{\omega} \mid i \in C_{1} \rightarrow T\left(C, \succ_{i}\right)=\{i\}\right\}
$$

Later we extend, however, the domain of voting procedures to the set of all logically possible profiles $\Sigma^{\omega}$. 
For voting procedures we require the following conditions:

Independence of non-voters' preferences: The outcome of a voting procedure depends only on the voters' preferences.

It is stated as item (ii) in [4].

Independence of irrelevant alternatives (IIA): For all $B \in \mathcal{B}, v(p, B)=$ $v\left(p^{\prime}, B\right)$ for all $p, p^{\prime} \in \Sigma^{\omega}(r)$ such that $\left.p\right|_{B}=\left.p^{\prime}\right|_{B}$.

It is stated as item (iii) in [4], and is called independence of infeasible alternatives in [5].

Next we define candidate stability and strong candidate stability.

Candidate stability: For all $i \in C$ and all $p \in \Sigma^{\omega}(r), v(p, C) \succ_{i} v(p, C \backslash\{i\})$ or $v(p, C)=v(p, C \backslash\{i\})$.

It means that each candidate prefers the outcome if all candidates are on the ballot to the outcome that would obtain if he withdrew from the election, or they are the same.

Strong candidate stability: For all $i \in C$ and all $p \in \Sigma^{\omega}(r)$, if $v(p, C) \neq i$, then $v(p, C)=v(p, C \backslash\{i\})$.

It requires that there be no change in the outcome of an election if a candidate withdraws who would lose if every candidate stood for office.

We define some other properties of voting procedures.

Unanimity: For all $B \in \mathcal{B}$ and all $p \in \Sigma^{\omega}(r)$, if $T\left(B, \succ_{i}\right)=\{x\}$ for all $i \in V$, then $v(p, B)=x$.

It requires that candidate $x$ is chosen when all voters most prefer $x$ in $B$. As stated in [5] if there are more than one voting candidates, unanimity is vacuous. Thus, we define the following condition.

Strong unanimity: For all $B \in \mathcal{B}$ and all $p \in \Sigma^{\omega}(r)$, if $T\left(B, \succ_{i}\right)=\{x\}$ for all $i \in V \backslash C_{1}$ and $T\left(B \backslash\{i\}, \succ_{i}\right)=\{x\}$ for all $i \in C_{1} \backslash\{x\}$, then $v(p, B)=x$. 
It requires that candidate $x$ is chosen when all voters most prefer $x$ in $B$ when each voting candidate's preference for himself (other than the preference of voter $x$ if $x$ is a voting candidate) is ignored.

Now we define a dictator of a voting procedure.

Dictator: If the outcome of a voting procedure on every $B \subset \mathcal{B}$ at every profile is always one of the top-ranked candidates of a particular voter at that profile, for example, voter $i$, that is, $v(p, B) \in T\left(B, \succ_{i}\right)$ at every profile $p \in \Sigma^{\omega}(r)$, then he is a dictator of the voting procedure. When a dictator is a voting candidate, the condition is that $v(p, B)=i$ if $i \in B$ and $v(p, B) \in T\left(B, \succ_{i}\right)$ if $i \notin B$ for $i \in C_{1}$ at every profile. But, we will show that a dictator is not a voting candidate.

We extend the domain of voting procedures to the set of all logically possible profiles $\Sigma^{\omega}$. Denote a profile in $\Sigma^{\omega}(r)$ by $p(r)$ and the preference of a voter $i$ by $\succ_{i}(r)$. We define the outcome of a voting procedure for $B \in \mathcal{B}$ at a profile $p$ in $\Sigma^{\omega} \backslash \Sigma^{\omega}(r)$ as follows:

$$
\begin{gathered}
v(p, B)=v(p(r), B) \text { such that }\left.\succ_{i}\right|_{B \backslash\{i\}}=\left.\succ_{i}(r)\right|_{B \backslash\{i\}} \text { for all } i \in C_{1} \\
\text { and }\left.\succ_{i}\right|_{B}=\left.\succ_{i}(r)\right|_{B} \text { for all } i \in V \backslash C_{1}
\end{gathered}
$$

It means that the outcome of a voting procedure ignores a change in the preference of every voting candidate about himself, and only a change in his preference about candidates excluding him can affect the outcome. By this definition any voting procedure is defined over unrestricted domain. We call this voting procedure an extended voting procedure.

About the relationship between original voting procedures and extended voting procedures we can show

Lemma 1. If the original voting procedures satisfy strong candidate stability, strong unanimity and IIA, then the extended voting procedures satisfy strong candidate stability, unanimity and IIA.

Proof. See Appendix A.

We define some properties of extended voting procedures. 
Weak Pareto optimality: For any ordered pair of candidates $(x, y)$ of every $B \in \mathcal{B}$ at every profile, if all voters prefer $x$ to $y\left(x \succ_{i} y\right.$ for all $\left.i \in V\right)$, then any voting procedure does not choose $y$.

Independence: Let $B$ be a set of candidates (a subset of $C)^{3}$. If profiles $p$ and $p^{\prime}$ agree on $B$, then $v(p, A)=v\left(p^{\prime}, A\right) \in B$, or at least one of $v(p, A)$ and $v\left(p^{\prime}, A\right)$ does not belong to $B$.

Monotonicity: For arbitrary two candidates $x$ and $y$, suppose that at a profile $p$ some voters prefer $x$ to $y$ and all other voters prefer $y$ to $x$, and a voting procedure chooses $x$. If voters, who prefer $x$ to $y$ at $p$, prefer $x$ to $y$ at another profile $p^{\prime}$, then the voting procedure does not choose $y$ at $p^{\prime}$ regardless of the preferences of other voters.

The condition of independence is similar to the analogous condition by [8] for social choice correspondences.

Clearly weak Pareto optimality implies unanimity for extended voting procedures. But, we will show that unanimity with strong candidate stability implies weak Pareto optimality.

Hereafter we abbreviate "extended" for voting procedures when it does not cause any confusion.

In Lemma 2 of [4] it was shown that if $C \cap V=\emptyset$, candidate stability implies strong candidate stability, and they proved the following two theorems.

Theorem 1 (Theorem 1 in [4]). If $C \cap V=\emptyset$ (there is no voting candidate), and a voting procedure is candidate stable and satisfies unanimity and IIA, then it is dictatorial.

Theorem 2 (Theorem 4 in [4]). If a voting procedure is strongly candidate stable and satisfies strong unanimity and IIA, then it is dictatorial and the dictator is in $V \backslash C_{1}$.

In [5] Theorem 1 is called the candidate stability theorem, and Theorem 2 is called the strong candidate stability theorem. Since Theorem 1 is proved as a corollary of Theorem 2, we will consider only Theorem 2.

${ }^{3} B$ is not necessarily a feasible set. It may contain only two candidates. 
As preliminary results, we show that a dictator is not a voting candidate, and that unanimity with strong candidate stability implies weak Pareto optimality in the following lemmas.

Lemma 2. A dictator, if it exists, is not a voting candidate.

Proof. Suppose that a dictator is a voting candidate $i$. Then, we always have $v(p, C)=i$ at every profile. Consider a profile such that

$$
T\left(C, \succ_{j}\right)=\{x\}, x \neq i \text { for all } j \in V \backslash\{i\} \text { and } T\left(C \backslash\{i\}, \succ_{i}\right)=\{x\} .
$$

Then, strong unanimity (or unanimity for extended voting procedures) means $v(p, C)=x$. It is a contradiction. Therefore, no voting candidate can be a dictator.

Lemma 3. Unanimity with strong candidate stability implies weak Pareto optimality.

Proof. See Appendix B.

Next we show independence and monotonicity in the following lemma.

Lemma 4. If a voting procedure satisfies strong candidate stability, weak Pareto optimality and IIA, then it satisfies independence and monotonicity.

Proof. See Appendix C.

We define two additional terms and show two lemmas.

Decisiveness: If, whenever all voters in a group $G$ prefer a candidate $x$ to another candidate $y$, a voting procedure does not choose $y$ regardless of the preferences of other voters, then $G$ is decisive for $x$ against $y$.

Decisive set: If a group $G$ is decisive about every pair of candidates, then it is called a decisive set.

The meaning of the word "decisive" is similar to the meaning of the same term used in [15] for binary social choice rules. A decisive set for a voting procedure may consist of one voter, then he is a dictator of the voting procedure.

Now we show the following lemma. 
Lemma 5. If a group $G$ is decisive for one candidate against another candidate, then it is a decisive set.

Proof. We present the proof of the case where there are more than three candidates. The proof of the case where there are only three candidates is similar.

Assume that $G$ is decisive for $x$ against $y$. Let $z$ and $w$ be arbitrary candidates other than $x$ and $y$. Consider the following profile.

1. Voters in $G$ prefer $z$ to $x$ to $y$ to $w$ to all other candidates.

2. Other voters prefer $y$ to $w$ to $z$ to $x$ to all other candidates.

The fact that $G$ is decisive for $x$ against $y$ and weak Pareto optimality imply that the voting procedure chooses $z$ at this profile. By monotonicity the voting procedure does not choose $w$ so long as the voters in $G$ prefer $z$ to $w$. It means that $G$ is decisive for $z$ against $w$. Based on this fact, by similar procedures we can show that $G$ is decisive for $z$ against $x$ (or $y$ ), for $y$ (or $x$ ) against $w$, and for $y$ against $x$. Since $z$ and $w$ are arbitrary, $G$ is a decisive set.

Next we can show the following result.

Lemma 6. If two groups $G$ and $G^{\prime}$, which are not disjoint, are decisive sets, then their intersection $G \cap G^{\prime}$ is also a decisive set.

Proof. Consider the following profile.

1. Voters in $G \backslash\left(G \cap G^{\prime}\right)$ prefer $z$ to $x$ to $y$ to all other candidates.

2. Voters in $G^{\prime} \backslash\left(G \cap G^{\prime}\right)$ prefer $y$ to $z$ to $x$ to all other candidates.

3. Voters in $G \cap G^{\prime}$ prefer $x$ to $y$ to $z$ to all other candidates.

4. Voters in $V \backslash\left(G \cup G^{\prime}\right)$ prefer $z$ to $y$ to $x$ to all other candidates.

Since $G$ and $G^{\prime}$ are decisive sets, the voting procedure chooses $x$. Since only voters in $G \cap G^{\prime}$ prefer $x$ to $z$ and all other voters prefer $z$ to $x$, monotonicity implies that $G \cap G^{\prime}$ is decisive for $x$ against $z$. By Lemma 5 it is a decisive set.

This lemma implies that the intersection of a finite number of decisive sets is also a decisive set. 


\section{An extended version of Cantor's diagonal argument and existence of dictator for voting procedures}

Consider profiles such that one voter (denoted by $i$ ) prefers $x$ to $y$ to $z$ to all other candidates, and all other voters prefer $z$ to $x$ to $y$ to all other candidates. Denote such a profile by $p^{i}$, and the set of such profiles for all $i$ by $\bar{\Sigma}^{\omega}$. By weak Pareto optimality any voting procedure chooses $x$ or $z$. If the voting procedure chooses $x$ at $p^{i}$ for some $i$, then by monotonicity individual $i$ is decisive for $x$ against $z$, and by Lemma 5 he is a dictator. On the other hand, if the voting procedure chooses $z$ at $p^{i}$ for all $i \in N$, then there exists no dictator, and a group $N \backslash\{i\}$ is a decisive set for all $i \in N$. By Lemma 6 in the latter case all co-finite sets (sets of individuals whose complements are finite sets) are decisive sets. Thus, we obtain the following theorem.

Theorem 3. Any strongly candidate stable voting procedure satisfying strong unanimity and IIA has a dictator or has no dictator, and in the latter case all co-finite sets are decisive sets.

An extended version of Cantor's theorem. According to [19] we present an extended version of Cantor's theorem and its proof.

Theorem 4. Let $Y$ be a set, and $\alpha: Y \rightarrow Y$ a function without a fixed point (for all $y \in Y, \alpha(y) \neq y$ ), $T$ and $S$ sets and $\beta: T \rightarrow S$ a function that is onto (i.e., has a right inverse $\bar{\beta}: S \rightarrow T$ ), then for all functions $f: T \times S \rightarrow Y$ the function $g: T \rightarrow Y$ constructed as follows

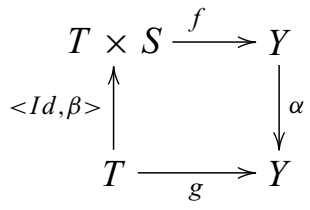

is not representable by $f$. Where Id is the identity mapping on $T$.

Proof. See Appendix D.

The famous Cantor's theorem is derived as a corollary of this theorem. 
Theorem 5. The cardinality of the power set $\mathcal{P}(\mathbb{N})$ of $\mathbb{N}$ is larger than the cardinality of $\mathbb{N}$, or in other words, there is no onto map $\mathbb{N} \rightarrow \mathcal{P}(\mathbb{N})$.

Proof. See Appendix E.

The following theorem is the main result of this paper.

Theorem 6. Whether or not a strongly candidate stable voting procedure has a dictator is not decidable, and this undecidability is proved using Cantor's diagonal argument.

Proof. Let $V$ be the set of strongly candidate stable voting procedures on the profiles in $\bar{\Sigma}^{\omega}$ defined above, $\mathbb{N}$ be the set of natural numbers, $I d$ be the identity mapping of $(V, \mathbb{N})$ and $\beta$ be a function such that $\beta:(V, \mathbb{N}) \rightarrow V$ that is onto. It is a projection function. Assume that there exists some program to decide whether or not a strongly candidate stable voting procedure has a dictator in finite steps. It reads the profiles in $\bar{\Sigma}^{\omega}$ from $p^{1}$ step by step. Denote such a program by $f$. Let $v_{1}$ and $v_{2}$ be two voting procedures in $V$ and $Y=\{0,1\}$. Then, $f$ is defined as follows:

$$
f:(V, \mathbb{N}) \times V \rightarrow Y:\left\{\begin{aligned}
f\left(v_{1}, N, v_{2}\right)= & 1 \text { if } f \text { judges that at least one of } \\
& v_{1} \text { and } v_{2} \text { has a dictator in at } \\
& \text { most } N \text { steps. } \\
f\left(v_{1}, N, v_{2}\right)= & 0 \text { if } f \text { judges that both } v_{1} \text { and } v_{2} \\
& \text { has no dictator in at most } N \text { steps. }
\end{aligned}\right.
$$

Define $\alpha$ as follows:

$$
\alpha: Y \rightarrow Y: \alpha(0)=1 \text { and } \alpha(1)=0
$$

$\alpha$ has no fixed point. We construct $g: V \times \mathbb{N} \rightarrow V$ as the following composition of three functions, $<I d, \beta>, f$ and $\alpha$.

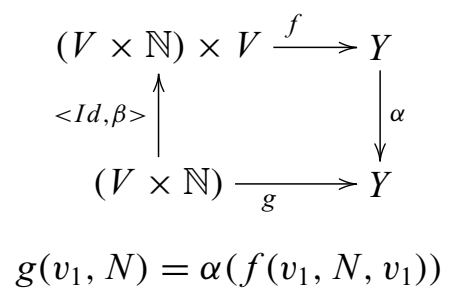


Let $g\left(v_{1}, N\right)=1$ mean that $v_{1}$ chooses $x$ at a profile $p^{N}$. Then, voter $N$ is a dictator of $v_{1} \cdot g\left(v_{1}, N\right)=0$ means that $v_{1}$ chooses $z$ at a profile $p^{N}$. Then, voter $N$ is not a dictator of $v_{1}$. When voter $N$ is a dictator of $v_{1}$, we have

$$
g\left(v_{1}, N\right)=f\left(v_{1}, N, v_{2}\right)=1
$$

for all $v_{2}$, that is, $g(-)$ is representable by $f\left(-, N, v_{2}\right)$ in the case where $g(-)=$ $f\left(-, N, v_{2}\right)=1$. We show that it leads to contradiction by similar arguments to those in the proof of Theorem 4. Evaluation at $v_{2}=v_{1}$ gives

$$
\begin{aligned}
f\left(v_{1}, N, v_{1}\right) & =g\left(v_{1}, N\right) \text { if voter } N \text { is a dictator of } v_{1} \\
& =\alpha\left(f\left(v_{1}, N, v_{1}\right)\right) \text { by definition of } g
\end{aligned}
$$

This means that $\alpha$ has a fixed point $f\left(v_{1}, N, v_{1}\right)$. It is a contradiction. Therefore, there does not exist any program to decide whether or not a strongly candidate stable voting procedure has a dictator in finite steps.

\section{Final Remark}

We have examined the strongly candidate stability theorem in an infinite society. We have shown that whether a strongly candidate stable voting procedure has a dictator or not is undecidable using Cantor's diagonal argument. The assumption of an infinite society seems to be unrealistic. But [11] presented an interpretation of an infinite society based on a finite number of individuals and a countably infinite number of uncertain states.

\section{Appendices}

\section{A Proof of Lemma 1}

First we show strong candidate stability. Let $p \in \Sigma^{\omega}, x \in C$, and assume $v(p, C) \neq x$. By definition $v(p, C)=v(p(r), C) \neq x$ for $p(r) \in \Sigma^{\omega}(r)$ such that $\left.\succ_{i}\right|_{C \backslash\{i\}}=\left.\succ_{i}(r)\right|_{C \backslash\{i\}}$ for all $i \in C_{1}$ and $\left.\succ_{i}\right|_{C}=\left.\succ_{i}(r)\right|_{C}$ for all $i \in$ $V \backslash C_{1}$. Strong candidate stability implies $v(p(r), C)=v(p(r), C \backslash\{x\})$. By definition we have $v(p, C \backslash\{x\})=v(p(r), C \backslash\{x\})$. Then, it follows $v(p, C)=v(p, C \backslash\{x\})$. Thus, the extended voting procedures satisfy strong candidate stability. 
Next consider unanimity. Let $p \in \Sigma^{\omega}, B \in \mathcal{B}$ and $x \in B$, and assume $T\left(B, \succ_{i}\right)=\{x\}$ for all $i \in V$. Then, we have $T\left(B, \succ_{i}(r)\right)=\{x\}$ for all $i \in V \backslash C_{1}$ and $T\left(B \backslash\{i\}, \succ_{i}(r)\right)=\{x\}$ for all $i \in C_{1} \backslash\{x\}$ at $p(r) \in \Sigma^{\omega}(r)$ such that $\left.\succ_{i}\right|_{B \backslash\{i\}}=\left.\succ_{i}(r)\right|_{B \backslash\{i\}}$ for all $i \in C_{1}$ and $\left.\succ_{i}\right|_{B}=\left.\succ_{i}(r)\right|_{B}$ for all $i \in V \backslash C_{1}$. By strong unanimity we have $v(p(r), B)=x$. Then, by definition $v(p, B)=x$. It means unanimity for the extended voting procedures.

Finally consider IIA. Let $B \in \mathcal{B}, p$ and $p^{\prime}$ be two profiles in $\Sigma^{\omega}$ such that $\left.p\right|_{B}=\left.p^{\prime}\right|_{B}$. Then, we have $\left.p(r)\right|_{B}=\left.p^{\prime}(r)\right|_{B}$ for $p(r)$ and $p^{\prime}(r)$ in $\Sigma^{\omega}(r)$ such that $\left.\succ_{i}\right|_{B \backslash\{i\}}=\left.\succ_{i}(r)\right|_{B \backslash\{i\}}$ and $\left.\succ_{i}^{\prime}\right|_{B \backslash\{i\}}=\left.\succ_{i}^{\prime}(r)\right|_{B \backslash\{i\}}$ for all $i \in C_{1}$ and $\left.\succ_{i}\right|_{B}=\left.\succ_{i}(r)\right|_{B},\left.\succ_{i}^{\prime}\right|_{B}=\left.\succ_{i}^{\prime}(r)\right|_{B}$ for all $i \in V \backslash C_{1}$. By IIA $v(p(r), B)=v\left(p^{\prime}(r), B\right)$. By definition $v(p, B)=v(p(r), B)$ and $v\left(p^{\prime}, B\right)=$ $v\left(p^{\prime}(r), B\right)$. Then, it follows $v(p, B)=v\left(p^{\prime}, B\right)$, and the extended voting procedures satisfy IIA.

\section{B Proof of Lemma 3}

(1) First we show weak Pareto optimality when $B=C$. Given a profile $p$ and a set of candidates $S$ we denote by $p * S$ the profile obtained by moving all candidates in $C \backslash S$ to the last position of the preferences of voters keeping their preferences about candidates in $C \backslash S$.

Assume that at a profile $p$ about a pair of candidates $(x, y)$ all voters prefer $x$ to $y$ and $f(p, C)=y$. Let us denote candidates other than $x$ and $y$ by $z_{1}, z_{2}, \cdots, z_{m-2}$, and consider a sequence of feasible sets $B_{1}=C \backslash\left\{z_{1}\right\}$, $B_{2}=C \backslash\left\{z_{2}\right\}, \cdots, B_{m-2}=C \backslash\left\{z_{m-2}\right\}$. Corresponding to this sequence, we construct a sequence of profiles by letting

$$
\begin{aligned}
& p_{1}=p * B_{1}, \\
& p_{2}=p_{1} * B_{1} \cap B_{2}, \cdots, \\
& p_{h}=p_{h-1} * \bigcap_{j=1,2, \cdots, h} B_{j}, \cdots, \\
& p_{m-2}=p_{m-3} * \bigcap_{j=1,2, \cdots, m-2} B_{j} .
\end{aligned}
$$

Since $f(p, C)=y$, it follows $f\left(p, B_{1}\right)=y$ by strong candidate stability; furthermore $f\left(p_{1}, B_{1}\right)=y$ by IIA. Strong candidate stability implies 
$f\left(p_{1}, C\right) \cap\left\{z_{1}, y\right\} \neq \emptyset$ because if $f\left(p_{1}, C\right) \neq y, f\left(p_{1}, C\right)$ must be $z_{1}$. Then, it follows $f\left(p_{1}, B_{2}\right) \cap\left\{z_{1}, y\right\} \neq \emptyset$ by strong candidate stability; furthermore $f\left(p_{2}, B_{2}\right) \cap\left\{z_{1}, y\right\} \neq \emptyset$ by IIA. Then, strong candidate stability implies $f\left(p_{2}, C\right) \cap\left\{z_{1}, z_{2}, y\right\} \neq \emptyset$. Repeated use of this argument leads to the conclusion $f\left(p_{m-2}, C\right) \cap\left\{z_{1}, z_{2}, \cdots, z_{m-2}, y\right\} \neq \emptyset$. At $p_{m-2}$ all voters most prefer $x$. Therefore, unanimity is violated.

(2) Next we consider the case where $|B|=m-1$. Let denote the candidate which is not included in $B$ by $z$. Assume that at a profile $p$ for a pair of candidates $(x, y)$ all voters prefer $x$ to $y$ and $f(p, B)=y$. Consider a profile $p^{\prime}$ which is constructed from $p$ by moving $z$ to the last position in each voter's preference. By IIA $f\left(p^{\prime}, B\right)=y$. Then, by strong candidate stability we have $f\left(p^{\prime}, C\right) \cap\{y, z\} \neq \emptyset$ because if $f\left(p^{\prime}, C\right) \neq y, f\left(p^{\prime}, C\right)$ must be $z$. But it contradicts the result of (1) because all voters prefer $x$ to $y$ and $z$ at $p^{\prime}$.

\section{Proof of Lemma 4}

(1) Independence: Assume that there exist two profiles $p, p^{\prime}$ and a set $B$ such that $p$ and $p^{\prime}$ agree on $B, x=f(p, C) \in B, y=f\left(p^{\prime}, C\right) \in B$ and $x \neq y$.

Denote $x=x_{m-1}$ and $y=x_{m}$, and let $B=\left\{x_{l}, x_{l+1}, \cdots, x_{m-1}, x_{m}\right\}$ where $1 \leqq l \leqq m-1$. Let us consider a profile $p^{\prime \prime}$ such that all voters prefer candidates in $B$ to $x_{1}$ to $x_{2}$ to $\cdots$ to $x_{l-1}$ and their preferences about candidates in $B$ are the same as those at $p$. There exists a sequence of subsets of $C, C_{1}, C_{2}, \cdots, C_{l-1}$ such that

$$
C_{h}=C \backslash\left\{x_{h}\right\} \text { and } \bigcap_{h=1,2, \cdots, l-1} C_{h}=B .
$$

Corresponding to this sequence we construct a sequence of profiles $p_{1}$, $p_{2}, \cdots, p_{l-1}$ as follows,

(a) at $p_{1}$ all voters prefer all candidates other than $x_{1}$ to $x_{1}$ and their preferences about candidates other than $x_{1}$ are the same as those at $p$, 
(b) at $p_{2}$ all voters prefer all candidates other than $x_{2}$ to $x_{2}$ and their preferences about candidates other than $x_{2}$ are the same as those at $p_{1}$,

and so on. Clearly $p_{l-1}=p^{\prime \prime}$. Now suppose $x_{m-1}=f\left(p_{h-1}, C\right)$. It follows $x_{m-1}=f\left(p_{h-1}, C_{h}\right)$ by strong candidate stability. Furthermore $x_{m-1}=f\left(p_{h}, C_{h}\right)$ by IIA. On the other hand, we have $f\left(p_{h}, C\right) \neq$ $x_{h}$ by weak Pareto optimality. Thus, strong candidate stability implies $x_{m-1}=f\left(p_{h}, C\right)$. Since $x_{m-1}=f(p, C)$, by induction it follows $x_{m-1}=f\left(p^{\prime \prime}, C\right)$.

Now consider the sequence of profiles starting from $p^{\prime}, p_{1}^{\prime}, p_{2}^{\prime}, \cdots, p_{l-1}^{\prime}$ such that at $p_{1}^{\prime}$ all voters prefer all candidates other than $x_{1}$ to $x_{1}$ and their preferences about candidates other than $x_{1}$ are the same as those at $p^{\prime}$, and so on. Clearly $p_{l-1}^{\prime}=p^{\prime \prime}$. By an argument similar to that in the case of profiles starting from $p$ we can show that $x_{m}=f\left(p^{\prime}, C\right)$ implies $x_{m}=f\left(p^{\prime \prime}, C\right)$. It is a contradiction. Thus, if $x=f(p, C) \in B, y \in B$ and $y \neq x$, then a candidate other than $y$ is chosen at $p^{\prime}$.

(2) Monotonicity: We use notations in the definition of monotonicity. Let $z$ be a given candidate other than $x$ and $y$.

Consider the following profiles.

(a) $p^{\prime \prime}$ : Voters, who prefer $x$ to $y$ at $p$, prefer $x$ to $y$ to $z$ to all other candidates. Voters, who prefer $y$ to $x$ at $p$, prefer $y$ to $z$ to $x$ to all other candidates.

(b) $p^{*}$ : Voters, who prefer $x$ to $y$ at $p$, prefer $x$ to $z$ to $y$ to all other candidates. Voters, who prefer $y$ to $x$ at $p, \operatorname{prefer} z$ to $x$ and prefer $z$ to $y$ to all other candidates (their preferences about $x$ and $y$ are not specified).

By independence (about $\{x, y\}$ ) and weak Pareto optimality the voting procedure chooses $x$ at $p^{\prime \prime}$. Again by independence (about $\{x, z\}$ ) and weak Pareto optimality the voting procedure chooses $x$ at $p^{*}$. Then, independence (about $\{x, y\}$ ) implies that the voting procedure does not choose $y$ so long as voters, who prefer $x$ to $y$ at $p$, prefer $x$ to $y$. 


\section{Proof of Theorem 4}

Let $Y, \alpha, T$ and $\beta$ be given. Let $\bar{\beta}: S \rightarrow T$ be the right inverse of $\beta$. By definition

$$
g(t)=\alpha(f(t, \beta(t)))
$$

We show that for all $s \in S g(-) \neq f(-, s)$. If $g(-)=f(-, s)$, then evaluation at $\bar{\beta}\left(s_{0}\right)$ gives

$$
\begin{aligned}
f\left(\bar{\beta}\left(s_{0}\right), s_{0}\right) & =g\left(\bar{\beta}\left(s_{0}\right)\right) \text { by representability of } g \\
& =\alpha\left(f\left(\bar{\beta}\left(s_{0}\right), \beta\left(\bar{\beta}\left(s_{0}\right)\right)\right)\right) \text { by definition of } g \\
& =\alpha\left(f\left(\bar{\beta}\left(s_{0}\right), s_{0}\right)\right) \text { by definition of right inverse }
\end{aligned}
$$

This means that $\alpha$ has a fixed point.

\section{E Proof of Theorem 5}

About $T, S, \beta$ and $\alpha$ in Theorem 4 we assume that $T=S=\mathbb{N}, \beta=I d$ be the identity mapping on $T, Y=\mathbf{2}=\{0,1\}$ and $\alpha$ be a function such that $\alpha(1)=0$ and $\alpha(0)=1$. Assume that there is an onto map $h: \mathbb{N} \rightarrow \mathcal{P}(\mathbb{N})$, and denote $h(n)=S_{h(n)} . S_{h(n)}$ is a subset of $\mathbb{N}$. Consider a function $f: \mathbf{N} \times \mathbf{N} \rightarrow \mathbf{2}$ such that $f(n, m)=1$ when $n \in S_{h(m)}$ and $f(n, m)=0$ when $n \notin S_{h(m)}$ for $n, m \in \mathbb{N}$. A function $g: \mathbb{N} \rightarrow \mathbf{2}$ is constructed as follows

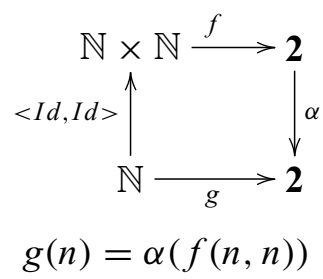

$g(n)$ is a characteristic function of the set

$$
G=\left\{n \mid n \notin S_{h(n)}\right\}
$$

Thus, $g(n)=1$ when $n \in G$ and $g(n)=0$ when $n \notin G$. Since $G$ is a subset of $\mathbb{N}$, we have $g(-)=f(-, m)$ for some $m \in \mathbb{N}$, that is, $g(-)$ must be representable by $f(-, m)$. But, since $\alpha$ has no fixed point, by Theorem $4 g(-)$ is not representable by $f(-, m)$. Therefore, there does not exist an onto map $h: \mathbb{N} \rightarrow \mathcal{P}(\mathbb{N})$. 
Acknowledgments. This research was partially supported by the Ministry of Education, Science, Sports and Culture, Grant-in-Aid, and the Zengin Foundation for Studies on Economics and Finance, Grant-in-Aid, in Japan.

\section{REFERENCES}

[1] K.J. Arrow, Social Choice and Individual Values, Second Edition. Yale University Press, 1963.

[2] P. Batteau, J.-M. Blin and B. Monjardet, Stability of aggregation procedures, ultrafilters and simple games. Econometrica, 49 (1981), 527-534.

[3] D. Bridges and F. Richman, Varieties of Constructive Mathematics (London Mathematical Society Lectures Notes Ser. Vol. 97). Cambridge University Press, 1987.

[4] B. Dutta, M.O. Jackson and M. Le Breton, Strategic candidacy and voting procedures. Econometrica, 69 (2001), 1013-1037.

[5] L. Ehlers and J.A. Weymark, Candidate stability and nonbinary choice. Economic Theory, 22 (2003), 233-243.

[6] P.C. Fishburn, Arrow's impossibility theorem: Concise proof and infinite voters. Journal of Economic Theory, 2 (1970), 103-106.

[7] A.F. Gibbard, Manipulation of voting schemes: A general result. Econometrica, 41 (1973), 587-601.

[8] B. Hansson, The existence of group preference functions. Public Choice, 28 (1976), 89-98.

[9] A.P. Kirman and D. Sondermann, Arrow's theorem, many Agents and invisible dictators. Journal of Economic Theory, 5 (1972), 267-277.

[10] W. Lawvere, Diagonal arguments and Cartesian closed categories, in "Category theory, homology theory and their applications, II”, Springer, Berlin, 1969, 134-145.

[11] R. Mihara, Arrow's theorem and Turing computability. Economic Theory, 10 (1997), 257-276.

[12] E. Muller and M.A. Satterthwaite, The Equivalence of strong positive association and strategy-proofness. Journal of Economic Theory, 14 (1975), 412-418.

[13] E.A. Pazner and E. Wesley, Stability of social choices in infinitely large societies. Journal of Economic Theory, 14 (1977), 252-262.

[14] M.A. Satterthwaite, Strategyproofness and Arrow's conditions: Existence and correspondence theorems for voting procedures and social welfare functions. Journal of Economic Theory, 10 (1975), 187-217.

[15] Sen Amartya, Collective Choice and Social Welfare. North-Holland, Amsterdam, 1979.

[16] K. Suzumura, Welfare economics beyond welfarist-consequentialism. Japanese Economic Review, 51 (2000), 1-32. 
[17] Y. Tanaka, The Gibbard-Satterthwaite theorem of social choice theory in an infinite society and LPO (Limited principle of omniscience). Applied Mathematics and Computation, 193 (2007), 475-481.

[18] A. Taylor, Social Choice and the Mathematics of Manipulation. Cambridge University Press, 2005.

[19] N.S. Yanofsky, A universal approach to self-referential paradoxes, incompleteness and fixed points. Bulletin of Symbolic Logic, 9 (2003), 362-386. 